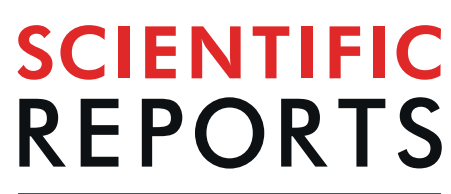

natureresearch

\title{
ROR $\gamma$ t may Influence the Microenvironment of Thyroid Cancer Predicting Favorable Prognosis
}

\author{
Lucas Leite Cunha ${ }^{1}$, Elaine Cristina Morari ${ }^{1,2}$, Suely Nonogaki ${ }^{3}$, Natassia Elena Bufalo ${ }^{1}$, \\ Ligia Vera Montalli da Assumpção ${ }^{1}$, Fernando Augusto Soares ${ }^{4,5}$, José Vassallo ${ }^{6}$ \& \\ Laura Sterian Ward ${ }^{1 *}$
}

We aimed to investigate the role of ROR $\gamma \mathrm{t}$ (Retinoic acid-related orphan receptor gamma) in the tumor microenvironment of differentiated thyroid carcinoma. We retrospectively analyzed 56 patients (48 papillary and 8 follicular thyroid carcinomas). Immunohistochemical expression of ROR $\gamma$ t was compared to other immune markers previously investigated by our group, clinical and pathological information. All patients presented cytoplasmic expression of ROR $\gamma t$ in thyroid tumor cells. Seven (12.5\%) patients presented no nuclear expression of ROR/t. Positivity was few (up to 10\%) in 14 patients; 10 to $50 \%$ in 5 patients (8.9\%); and more than $50 \%$ in 30 patients (53.6\%). Nuclear ROR $\gamma$ t positivity was associated with absence of distant metastasis at diagnosis $(p=0.013)$ and the need of less cumulative doses of radioactive iodine $(p=0.039)$. Patients whose tumors were positive for nuclear ROR $\gamma$ t presented higher 10 -years relapse-free survival rate than those patients who were negative for ROR $\gamma \mathrm{t}(\mathrm{p}=\mathbf{0 . 0 2 3}$ ). We classified the patients according to the clustering of immunological immunohistochemical markers. We were able to distinguish a subset $(A)$ of 38 patients who presented high expression of nuclear ROR $\gamma t$ and tended to be scarce in proinflammatory immune markers. Other 16 patients integrated a second subset (B) whose tumor microenvironment accumulated proinflammatory markers and presented low expression of nuclear nuclear ROR $\gamma t$. Distant metastasis at diagnosis were more frequent among patients from cluster $B$ than from cluster $A(p=0.008)$. Our results reinforce that the expression of $R O R \gamma t$ together with other immune markers might help predict the prognosis of patients with thyroid cancer and help individualize clinical management.

The American Cancer Society's most recent estimates for thyroid cancer in 2019 are about 52,070 new cases $(14,260 \text { in men and } 37,810 \text { in women })^{1}$. It is the most rapidly increasing cancer in the USA, tripling in the past three decades ${ }^{1}$. However, mortality rate has been steady for many years, and remains very low compared with most other cancers, suggesting widespread overdiagnosis (detection of tumors that will not cause clinical illness or death $)^{2-4}$. Current guidelines suggest that the patients with thyroid cancer should be treated according to their overall prognosis, their risk of recurrence and mortality, the treatment's benefits and possible troubles, and the patient's setting and values. Unfortunately, thyroid cancer will still be responsible for about 2,170 deaths $(1,020$ men and 1,150 women) in the USA during $2019^{1}$. A series of molecular markers have been actively investigated aiming to delineate patients who would benefit of a less or of a more aggressive management, and some of them are proven to be clinically useful and are increasingly used as adjunct diagnostic molecular tests with thyroid biopsy $^{5}$. Unfortunately, we still lack affordable, easy to perform tests capable to distinguish the low risk or indolent thyroid tumor from the one that will evolve and eventually kill the patient.

\footnotetext{
${ }^{1}$ Laboratory of Cancer Molecular Genetics, Faculty of Medical Sciences, University of Campinas (Unicamp), Campinas, Brazil. ²Department of Biological and Health Sciences, State University of Roraima, Boa Vista, Brazil. ${ }^{3}$ Adolfo Lutz Institute, São Paulo, Brazil. ${ }^{4}$ Department of General Pathology, Dental School, University of São Paulo, São Paulo, Brazil. ${ }^{5}$ Pathology Division, ID’Or Research Institute, Rede D’Or Hospitals Network, São Paulo, Brazil. ${ }^{6}$ Laboratory of Investigative and Molecular Pathology (Ciped), Faculty of Medical Sciences - University of Campinas (Unicamp), Campinas, Brazil. *email: ward@fcm.unicamp.br
} 
Inflammation is currently a hallmark of cancer and is known as an essential component of malignancies ${ }^{6}$. In fact, the tumor microenvironment is enriched with activated immune cells, which secrete cytokines and chemokines that trigger the expansion of both tumor and stromal cells ${ }^{7,8}$. Experimental studies have demonstrated that thyroid cancer cells express chemokine receptors, suggesting that local secretion of immune molecules may negatively influence the tumor behavior through proangiogenetic, cytoproliferative, and pro-metastatic effects ${ }^{9,10}$. On the other hand, the proliferation and activation of immune cells may stimulate the recognition of tumor specific antigens expressed by cancer cells leading to the tumor elimination (immunosurveillance) ${ }^{7}$. Tumor microenvironment inflammatory profile is closely related to the biological behavior of many tumors and our group has contributed to the knowledge in this field demonstrating that some cytokines may be associated to thyroid tumors features of aggressiveness and patients' outcome $e^{11,12}$.

The expression of retinoic acid-related orphan receptors have been implicated in a series of diseases, with both positive and negative impact depending on conditions that include a series of factors such as autoimmunity, inflammation, metabolic syndrome, neurological disorders, and cancer. ROR $\gamma \mathrm{t}$ (RAR-related orphan receptor gamma $\mathrm{T}$ ) is a transcription factor considered essential for lymphoid organogenesis ${ }^{13}$. ROR $\gamma$ t regulates the thymopoiesis by reducing apoptosis of thymocytes and inducing thymocyte differentiation into pro-inflammatory $\mathrm{T}$ helper 17 (Th17) cells ${ }^{14,15}$. In tumor biology, ROR $\gamma$ t has been associated to the promotion of lymphatic dissemination in breast cancer ${ }^{16}$. A recent investigation suggested that the expression of ROR $\gamma t$ in the tumor microenvironment of papillary thyroid cancer (PTC) patients could inhibit lymph node metastasis ${ }^{17}$. This finding may be of great clinical importance for the best management of PTC patients. In addition, a series of studies have proposed that antagonist of the ROR $\gamma$ receptor has therapeutic proprieties in inflammatory diseases ${ }^{18,19}$, and a number of synthetic ROR $\gamma$ receptor antagonists have been developed ${ }^{20}$. ROR $\gamma$ agonists (such as LYC-55716) may allow the immune system to combat cancer and some compounds are in clinical trials on patients with solid tumors ${ }^{21,22}$.

This study aimed to better understand the role of $\mathrm{ROR} \gamma \mathrm{t}$ in thyroid tumors microenvironment and its potential clinical utility.

\section{Material and Methods}

Patients. We retrospectively investigated consecutive patients with thyroid cancer who attended to our Thyroid Unit. Consent was obtained from each patient after full explanation of the purpose and nature of all procedures used. The Research Ethics Committee of our Hospital (A.C. Camargo Cancer Center, São Paulo, Brazil) approved our investigation. All research was performed in accordance with relevant guidelines/regulations. Written informed consent was obtained from all participants and/or their legal guardians. We excluded those patients with no histopathological confirmation of thyroid carcinoma, limited or no clinical information on their charts. We included 56 patients: 48 with papillary thyroid carcinoma (18 classic PTC, 15 follicular variant of PTC, 8 tall cells PTC and 7 poorly differentiated PTC) and 8 with follicular thyroid carcinoma (FTC). Fifty-two patients (44 with PTC and 8 with FTC) had sufficient and satisfactory tissue sample in our biobank available for molecular scrutiny of all immune markers.

Formalin-fixed paraffin-embedded tissues from all patients were reviewed for investigation of concurrent chronic lymphocytic thyroiditis (CLT) in nonmalignant thyroid parenchyma of the tumor contralateral lobe. Concurrent thyroiditis was histologically diagnosed by lymphocytic infiltration with lymphoid follicles and follicular regenerative activity with several small follicles, lined by Hurthle cells and fibrotic tissue ${ }^{23}$.

We classified as poorly differentiated PTC tumors with solid, trabecular or insular growth patterns, with no nuclear features of PTC and with one of the following features: convoluted nuclei, 3 or more mitotic figures per 10 high power field, and tumor necrosis ${ }^{24-26}$.

We evaluated presence/absence of both microscopic and gross extrathyroidal invasion. Microscopically, we considered capsular invasion and angio-lymphatic invasion. In order to stage patients, extrathyroidal extension was defined according to AJCC $8^{\text {th }}$ edition. Tumors with extrathyroidal extension were all of those in which gross extrathyroidal extension invaded only strap muscles from a tumor of any size.

Among the follicular variant of PTC cases, there were 4 patients presenting the infiltrative subtype and 11 patients presenting the encapsulated subtype. Among the FTC cases, we included 2 patients with minimally invasive FTC and 6 patients with widely invasive FTC. Five patients presented a unifocal lesion, while multifocality was found in $3 / 8$ patients. Gross extrathyroid extension was observed in one patient; microscopically vascular invasion was observed in $6 / 8$ patients. None of the patients with FTC presented lymph-node metastasis at diagnosis. Distant metastasis was observed in $3 / 8$ patients. Three patients presented oxyphilic cells in tumor parenchyma and one patient presented trabecular/insular growth pattern.

We used pTNM classification system (AJCC Cancer Staging Manual $8^{\text {th }}$ edition) for thyroid cancer as criteria of aggressiveness at diagnosis ${ }^{27}$. Patients were followed-up by standard protocol that include periodic total body scans, serum thyroglobulin and thyroid-stimulating hormone measurements, radiologic scrutiny (X-ray, ultrasonography, computed tomography scan) and other approaches to detect local or distant metastasis for a period of 16-322 months ( $157.7 \pm 68.3$ months). During the follow-up, patients presenting elevated non-stimulated serum thyroglobulin levels $(>2 \mathrm{mg} / \mathrm{dl})$ were submitted to a meticulous image search. The mentioned clinical parameters were used to define tumors as persistent/recurrent and/or presenting long distance metastasis. Patients were considered free-of disease when they evolved with stable unstimulated serum thyroglobulin levels $<2 \mathrm{ng} / \mathrm{dl}$ or undetectable thyroglobulin levels for more than 2 years after tumor resection, without any suspicion of recurrence ${ }^{28}$. We considered 42 patients free-of-disease and 14 presented recurrence/metastasis during the observation period. Two patients from our cohort died due to thyroid cancer during follow-up.

Immunohistochemistry and semiquantification. Samples from all specimens were reviewed with the intention to sort the most representative area designed to build a tissue microarray (Beecher Instruments ${ }^{\circledR}$, Silver Springs, MD, USA) for immunohistochemical analysis. Then, we obtained 4 tumor tissue cores from each patient: 

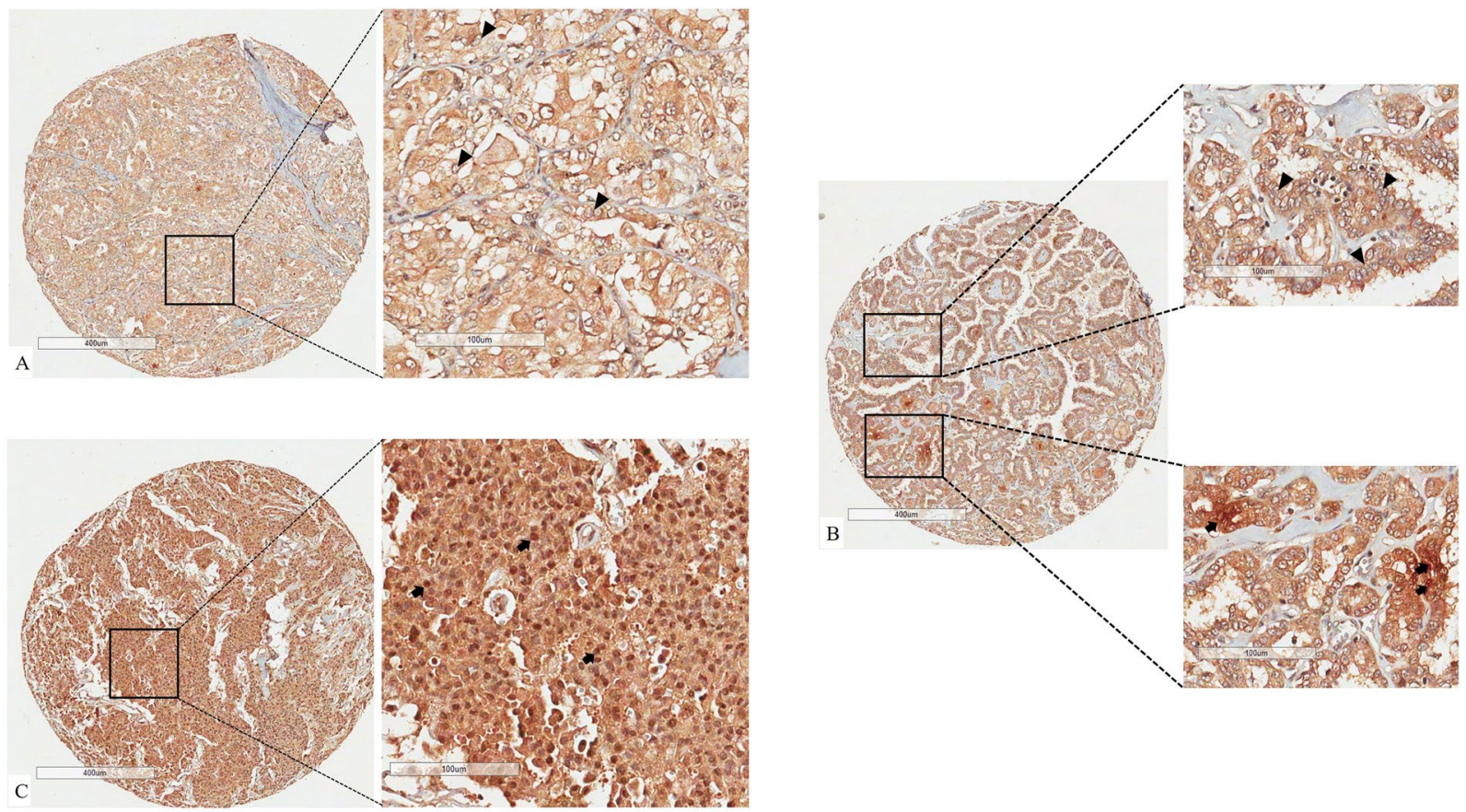

Figure 1. Different percentages of nuclear ROR $\gamma \mathrm{t}$ positivity. Panel (A) shows the diffuse brownish in cytoplasm but not in nuclear of thyroid carcinoma cells. Panel (B) evidence that major of nuclei of thyroid cancer cells are negative for ROR $\gamma$ t expression (upper right panel). Lower right panel shows a detail of focus of nuclei positive for ROR $\gamma$ t. Panel C represent a tissue spot in which all nuclei of thyroid cancer cells are positive for ROR $\gamma$ t. Black arrows point to nuclei positive for ROR $\gamma$ t. Black arrow head point to nucleai negative for ROR $\gamma$ t. Stromal components like collagen, vessels and myocytes are also not expected to express nuclear ROR $\gamma \mathrm{t}$, giving us a negative control for each spot analyzed.

2 spots were selected from representative area enriched with leukocytes and 2 other spots from representative area free of leukocytes. The use of two spots has been widely used in pathology and has shown good correlation to whole sections analyses.

We investigated the expression of IL-1 $\beta$, IL-17, IL-23, IL-10 and COX2 in tumor cells. We looked for intratumoral infiltration of immune cells using CD3, CD4, CD8, CD20, CD68, CD16 and CD45RO markers. Activated immune cells were analyzed using CD25, CD69 and Granzyme B markers. Additionally, we studied the expression of ROR $\gamma \mathrm{t}$ in tumors cells (Fig. 1). Immunohistochemistry was performed as previously described, using positive and negative controls in the same batch of reactions ${ }^{29}$. We used human reactive lymph node as positive control. Negative control was obtained performing the same batch of reaction in the same reactive lymph node, but, without adding primary antibody.

Semiquantification was performed by two investigators (LLC and ECM), as routinely done by our group ${ }^{30-32}$. Two experienced pathologists (JV and FAS) blinded to tumor information evaluated all slides. Cells were defined as positive for immunohistochemical markers when a clear cut brown staining was observed in the corresponding cellular localization. Visual analysis of aforementioned markers was done considering an approximate area of 0.79 $\mathrm{mm}^{2}$ per tissue microarray spot. We estimated the markers of tumor cells by the percentage of positive tumor cells and the intensity of its staining. We assessed the infiltration of immune cells classifying them as 0 (no immune cells infiltration), 1 (infiltration of up to 10 immune cells) and 2 (infiltration of more than 10 immune cells in each spot). ROR $\gamma$ t was cytoplasmic expressed in tumor cells from virtually all samples. Then, we categorized the patients according to the percentage of tumor cells harboring nuclear expression of ROR $\gamma$ t. Patients were classified as 0 (no tumor cells with nuclear expression of ROR $\gamma \mathrm{t}$ ), 1 ( 1 to $10 \%$ of tumor cells positive for nuclear expression of ROR $\gamma \mathrm{t}$ ), 2 (11 to 50\% of tumor cells positive for nuclear expression of ROR $\gamma \mathrm{t}$ ) and 3 (more than $50 \%$ of tumor cells positive for nuclear expression of ROR $\gamma \mathrm{t}$ ).

Cluster and statistical analysis. We performed the hierarchical cluster analysis of our immunohistochemical data. Cluster 3.0 was used to assess similarities/distances of patients according to the assembled immunological results. Following the instruction of the authors, the eight parameters (ROR $\gamma \mathrm{t}, \mathrm{CD} 8, \mathrm{CD} 16, \mathrm{COX} 2, \mathrm{IL}-1 \beta$, IL-17A, IL-10 and IL-23) were submitted to complete-linkage hierarchical clustering. Java TreeView was used to graphically construct the dendrogram that express similarities/distance of patients.

Statistical analysis was performed using the Statistical Package for the Social Sciences (SPSS) ${ }^{\circledR}$ software, version 23.0. First relapse was defined as end-point. Kaplan-Meier method was used to calculate relapse-free survival rates and survival were compared using log-rank test. Chi-square or Fisher's exact test were assessed to perform nonparametric analysis, when indicated. Mann-Whitney tests were used to compare continuous measures between two independent groups whose variables did not fit to normal distribution; Kruskal-Wallis test was 
performed to compare continuous measures between three or more groups whose variables did not fit normal distribution. All tests were conducted at a 0.05 significance level.

Compliance with ethical standards. Informed consent was obtained from each patient after full explanation of the purpose and nature of all procedures used. The Research Ethics Committee of our Hospital (A.C. Camargo Cancer Center, São Paulo, Brazil) approved our investigation

\section{Results}

Our cohort included 46 female and 10 male patients. Mean age at diagnosis was $42 \pm 17.3$ years; mean tumor size was $3.1 \pm 2.2 \mathrm{~cm}$. Twenty-eight patients (50\%) presented multifocality. Eleven patients $(20 \%)$ presented capsular invasion, $13(23.6 \%)$ presented angio-lymphatic invasion, $14(25.5 \%)$ presented extrathyroid invasion and 30 (54.4\%) presented capsular invasion, vascular invasion or gross extrathyroidal invasion at the diagnosis. Fourteen (25\%) patients had evidence of concurrent thyroiditis. Twenty-three $(41.8 \%)$ patients presented lymph-node involvement at diagnosis. Nine (16.1\%) patients presented long distant metastasis at diagnosis. The majority of patients were diagnosed in early stage of the disease (pTNM I; $n=28 ; 50 \%)$, whereas $13(23.2 \%)$ patients were diagnosed in pTNM II; 7 (12.5\%) patients were diagnosed in pTNM III; and 8 (14.3\%) patients were diagnosed in pTNM IV. Mean cumulative dose of ${ }^{131} \mathrm{I}$-iodine therapy was $269 \mathrm{mCi}( \pm 260 \mathrm{mCi}$, ranging from 0 to $1200 \mathrm{mCi})$. Table 1 summarizes the correlation between clinic and pathological features of patients with thyroid cancer and different tumor sub-types.

Among the patients with gross extrathyroidal extension, $84.6 \%$ of them also presented lymph-node metastasis at diagnosis $(\mathrm{p}=0.001)$, and $23.1 \%$ of them presented distant metastasis at diagnosis $(\mathrm{p}=0.027)$. Patients with PTC and gross extrathyroidal extension presented a poor prognosis compared with those with no gross extrathyroidal extension at the diagnosis $(46,2 \%$ of five-years survival rate versus $88 \%$, respectively; p-value $=0.001)$, reinforcing that gross extrathyroidal extension is associated with PTC aggressiveness.

All patients presented cytoplasmic expression of ROR $\gamma \mathrm{t}$ in thyroid tumor cells. Since cytoplasm is not the natural subcellular location of ROR $\gamma \mathrm{t}$, we investigated its nuclear expression by semiquantitative estimation of nuclear ROR $\gamma$ t positivity in tumor cells, as demonstrated in Fig. 1. Seven (12.5\%) patients presented no nuclear expression of ROR $\gamma \mathrm{t} ; 14$ patients (25.0\%) presented few ( 1 to $10 \%$ ) nuclear positivity; 5 patients (8.9\%) presented 11 to $50 \%$ of positivity; and 30 patients $(53.6 \%)$ presented more than $50 \%$ of positivity. Table 2 shows the association between nuclear expression of ROR $\gamma \mathrm{t}$ and clinical features of the patients. We found an association between ROR $\gamma t$ positivity and absence of distant metastasis at diagnosis $(p=0.013)$. Patients whose tumors were positive for nuclear ROR $\gamma t$ required less cumulative doses of radioactive iodine (RAI; $p=0.039$ ). We did not observe any difference neither concerning relapse-free survival nor in overall survival regarding ROR $\gamma$ t positivity. In order to accurately assess the prognostic impact of $\mathrm{ROR} \gamma \mathrm{t}$, we gathered patients with $1-10 \%$ of nuclear positivity (14 patients), patients with $11-50 \%$ of nuclear positivity (5 patients) and patients who presented more than $50 \%$ of nuclear positivity (30 patients) which were compared with patients whose tumors presented no nuclear positivity for ROR $\gamma$ t. Patients whose tumors were positive for nuclear ROR $\gamma t$ presented higher 10-years relapse-free survival rate $(79.3 \%)$ than those patients who were negative for ROR $\gamma t(42.9 \% ; p=0.023$; Fig. 2$)$.

We observed IL-17A expression in cytoplasm of thyroid cancer cells from all patients. Interestingly, 42 patients (75.0\%) presented a faint cytoplasmic expression of IL-17A, while strong diffuse expression was observed in the remaining 14 patients $(25.0 \%)$. We, then, investigated association between clinical data and IL-17A, considering faint versus strong IL-17A expression. IL-17A failed to be associated with clinical and pathological characteristics of aggressiveness.

Thirty-two patients (58.1\%) presented expression of IL-1 $\beta$, all of them in cytoplasmic subcellular location; twenty-three patients $(41.8 \%)$ presented no expression of IL-1 $\beta$. One patient presented a doubtful expression and was excluded from further analysis. Table 3 summarizes associations between IL- $1 \beta$ and clinical and pathological features of patients. IL-1 $\beta$ expression was observed more frequently among patients who presented no evidence of concurrent CLT $(\mathrm{p}=0.048)$, with distant metastasis at diagnosis $(\mathrm{p}=0.041)$. In addition, patients whose tumors presented IL-1 $\beta$ expression were diagnosed with larger tumor size $(p=0.032)$ and required a higher RAI cumulative doses $(p=0.004)$. Patients whose tumors were positive for IL- $1 \beta$ tended to present lower 10 -years relapse-free survival rate $(65.3 \%)$ than patients who were negative for IL-1 $\beta$ (87.0\%), but this difference was marginally significant $(\mathrm{p}=0.081)$.

Thirty-nine patients (69.6\%) presented cytoplasmic homogeneous expression of IL-23; the remaining 17 patients presented no expression of IL-23. As seen in Table 4, expression of IL-23 was more frequently found in patients who presented distant metastasis at diagnosis $(\mathrm{p}=0.031)$. Patients whose tumors presented IL-23 expression were diagnosed with larger tumor size $(\mathrm{p}=0.009)$ and required a higher RAI cumulative doses $(\mathrm{p}=0.037)$. Log-rank test did not evidence the expression of IL-23 as predictor of relapse-free survival (data not shown).

We compared nuclear ROR $\gamma$ t, IL-17A, IL-1 $\beta$ and IL23 expression with other immune markers previously investigated by our group ${ }^{31}$ (Table 5). Nuclear ROR $\gamma$ t was inversely associated with infiltration of CD16 lymphocytes $(p=0.047)$. Likewise, nuclear $R O R \gamma t$ expression was inversely correlated to COX2 $(p=0.024)$, IL-1 $\beta(p=0.001)$ and IL23 ( $p=0.043)$. IL-1 $\beta$ was directly associated with infiltration of CD $8+$ lymphocytes $(\mathrm{p}=0.002)$, infiltration of CD16 + lymphocytes $(\mathrm{p}=0.046)$, expression of COX2 $(\mathrm{p}<0.001)$, IL-10 $(\mathrm{p}=0.032)$ and IL23 ( $p<0.001)$. IL-23 was directly associated with infiltration of CD8 + lymphocytes $(\mathrm{p}=0.004)$, infiltration of CD16 + lymphocytes $(\mathrm{p}=0.003)$ and expression of COX2 $(\mathrm{p}<0.001)$. Figure 3 shows the immunostaining for the investigated interleukins.

After accessing the association of immunological markers with each other, we classified the patients according to the clustering of the significant markers (ROR $\gamma \mathrm{t}, \mathrm{CD} 8$, CD16, COX2, IL-1 $\beta$, IL-17A, IL-10 and IL-23). The complete immunohistochemical characterization of the eight variables was available for 54 patients. Figure 4 shows the dendrogram obtained after cluster immunological markers. Thirty-eight patients were categorized in 


\begin{tabular}{|c|c|c|c|c|c|c|c|c|}
\hline & Classic PTC & FV-PTC & TC-PTC & PD-PTC & FTC & p-value* & p-value** & p-value $* * *$ \\
\hline \multicolumn{9}{|l|}{ Gender } \\
\hline Female & $14(77.8)$ & $12(80.0)$ & $7(87.5)$ & $7(100.0)$ & $6(75.0)$ & 0.692 & 0.568 & 0.569 \\
\hline Male & $4(22.2)$ & $3(20.0)$ & $1(12.5)$ & $0(0.0)$ & $2(25.0)$ & & & \\
\hline $\begin{array}{l}\text { Age, Mean } \\
\pm \text { SD }\end{array}$ & $33.7 \pm 11.8$ & $43.1 \pm 17.3$ & $51.4 \pm 15.2$ & $38.3 \pm 19.7$ & $58.5 \pm 16.3$ & 0.006 & 0.056 & 0.022 \\
\hline \multicolumn{9}{|l|}{ Multifocality } \\
\hline Unifocal & $9(50.0)$ & $7(46.7)$ & $4(50.0)$ & $3(42.9)$ & $5(62.5)$ & 0.950 & 0.988 & 0.445 \\
\hline Multifocal & $9(50.0)$ & $8(53.3)$ & $4(50.0)$ & $4(57.1)$ & $3(37.5)$ & & & \\
\hline \multicolumn{9}{|c|}{ Concurrent CLT } \\
\hline Absent & $13(72.2)$ & $11(73.3)$ & $6(75.0)$ & $6(85.7)$ & $6(75.0)$ & 0.971 & 0.913 & 1.000 \\
\hline Present & $5(27.8)$ & $4(26.7)$ & $2(25.0)$ & $1(14.3)$ & $2(25.0)$ & & & \\
\hline \multicolumn{9}{|l|}{ pTNM } \\
\hline $\mathrm{I}$ & $10(55.6)$ & $9(60.0)$ & $2(25.0)$ & $6(85.7)$ & $1(12.5)$ & 0.035 & 0.025 & 0.073 \\
\hline II & $4(22.2)$ & $4(26.7)$ & $1(12.5)$ & $1(14.3)$ & $3(37.5)$ & & & \\
\hline III & $3(16.7)$ & $2(13.3)$ & $1(12.5)$ & $0(0.0)$ & $1(12.5)$ & & & \\
\hline IV & $1(5.6)$ & $0(0.0)$ & $4(50.0)$ & $0(0.0)$ & $3(37.5)$ & & & \\
\hline \multicolumn{9}{|c|}{ Lymph-node metastasis at diagnosis } \\
\hline Absent & $7(38.9)$ & $10(66.7)$ & $3(37.5)$ & $5(71.4)$ & $8(100.0)$ & 0.028 & 0.234 & 0.011 \\
\hline Present & $11(61.1)$ & $5(33.3)$ & $5(62.5)$ & $2(28.6)$ & $0(0.0)$ & & & \\
\hline \multicolumn{9}{|c|}{ Distant metastasis at diagnosis } \\
\hline Absent & $16(88.9)$ & $15(100.0)$ & $6(75.5)$ & $6(87.5)$ & $5(62.5)$ & 0.140 & 0.297 & 0.043 \\
\hline Present & $2(11.1)$ & $0(0.0)$ & $2(25.5)$ & $1(14.3)$ & $3(37.5)$ & & & \\
\hline \multicolumn{9}{|c|}{ Capsular invasion } \\
\hline Absent & $17(94.4)$ & $11(73.3)$ & $7(87.5)$ & $6(85.7)$ & $4(50.0)$ & 0.114 & 0.429 & 0.022 \\
\hline Present & $1(5.6)$ & $4(26.7)$ & $1(12.5)$ & $1(14.3)$ & $4(50.0)$ & & & \\
\hline \multicolumn{9}{|c|}{ Vascular invasion } \\
\hline Absent & $15(83.3)$ & $13(86.7)$ & $5(62.5)$ & $7(100.0)$ & $3(37.5)$ & 0.027 & 0.266 & 0.005 \\
\hline Present & $3(16.7)$ & $2(13.3)$ & $3(37.5)$ & $0(0.0)$ & $5(62.5)$ & & & \\
\hline \multicolumn{9}{|c|}{ Gross extrathyroid extension } \\
\hline Absent & $14(77.8)$ & $15(100.0)$ & $1(12.5)$ & $5(71.4)$ & $7(87.5)$ & $<0.001$ & $<0.001$ & 0.363 \\
\hline Present & $4(22.2)$ & $0(0.0)$ & $7(87.5)$ & $2(28.6)$ & $1(12.5)$ & & & \\
\hline \multicolumn{9}{|c|}{ Any invasion } \\
\hline Absent & $12(66.7)$ & $10(66.7)$ & $1(12.5)$ & $3(42.9)$ & $0(0.0)$ & 0.004 & 0.055 & 0.005 \\
\hline Present & $6(33.3)$ & $5(33.3)$ & $7(87.5)$ & $4(57.1)$ & $8(100.0)$ & & & \\
\hline $\begin{array}{l}\text { RAI doses, } \\
\text { Mean } \pm \text { SD }\end{array}$ & $220.4 \pm 173.8$ & $268.0 \pm 302.1$ & $406.2 \pm 322.3$ & $142.8 \pm 142.7$ & $356.2 \pm 323.4$ & 0.201 & 0.123 & 0.548 \\
\hline
\end{tabular}

Table 1. Correlation between clinic and pathological features of patients with thyroid cancer and different tumor sub-types. Abbreviations: SD, standard deviation; CLT, chronic lymphocytic thyroiditis; PTC, papillary thyroid carcinoma; FV-PTC, follicular variant of PTC; TC-PTC, tall cell variant of PTC; PD-PTC, poorly differentiated, PTC; FTC, follicular thyroid carcinoma; RAI, radioactive iodine therapy cumulative doses. Note: *comparison considering all different histologic subtypes (5 categories); **comparison considering different subtype of PTC (4 categories); ***comparison considering PTC versus FTC.

the first subset of subjects (cluster A) and most of them presented nuclear expression of ROR $\gamma$ t. Sixteen patients were categorized in the second subset of subjects (cluster B). Different tumor types were not associated to molecular cluster (Table 6). Distant metastasis at diagnosis was more frequently found among patients from cluster B $(37.5 \%)$ than among patients from cluster A $(7.9 \% ; \mathrm{p}=0.008)$. Patients from cluster A presented higher 10-years relapse-free survival rate $(78.5 \%)$ than those patients from cluster $B(62.5 \% ; p=0.185)$, but the difference was not significant.

\section{Discussion}

Our results showed that nuclear ROR $\gamma \mathrm{t}$ expression was associated to absence of distant metastasis at diagnosis and lower requirement of therapeutic RAI dose. Besides identifying less aggressive tumors, nuclear ROR $\gamma \mathrm{t}$ expression might predict favorable outcome in patients with thyroid carcinoma. Nuclear ROR $\gamma \mathrm{t}$ was negatively correlated to expression of IL-17A, IL-1 $\beta$ and IL-23, all cytokines engaged in promotion of Th17 related immune response. Interestingly, on the contrary of nuclear ROR $\gamma$ t expression, both IL-1 $\beta$ and IL-23 were correlated to more aggressive clinic and pathological features that included large tumor size, presence of distant metastasis at diagnosis and requirement of higher cumulative dose of RAI. In fact, when we gather the molecular markers through hierarchical cluster, patients whose tumor microenvironment was enriched with pro-inflammatory 


\begin{tabular}{|c|c|c|c|c|c|}
\hline \multirow[b]{2}{*}{ Clinical feature } & \multicolumn{4}{|c|}{ Estimation of percentage of tumor cells expressing nuclear ROR $\gamma \mathrm{t}$} & \multirow[b]{2}{*}{ p-value ${ }^{a}$} \\
\hline & $0 \%$ & $1-10 \%$ & $11-50 \%$ & $>50 \%$ & \\
\hline \multicolumn{6}{|l|}{ Gender } \\
\hline Male & $1(10.0)$ & $2(20.0)$ & $1(10.0)$ & $6(60.0)$ & 0.961 \\
\hline Female & $6(13.0)$ & $12(26.1)$ & $4(8.7)$ & $24(52.2)$ & \\
\hline \multicolumn{6}{|l|}{ Age at diagnosis } \\
\hline $45 \mathrm{yrs}$ or less & $3(11.1)$ & $9(33.3)$ & $2(7.4)$ & $13(48.1)$ & 0.583 \\
\hline More $45 \mathrm{yrs}$ & $4(13.8)$ & $5(17.2)$ & $3(10.3)$ & $17(58.6)$ & \\
\hline \multicolumn{6}{|l|}{ Histologic types } \\
\hline Classic PTC & $2(11.1)$ & $8(44.4)$ & $0(0.0)$ & $8(44.4)$ & $0.381 *$ \\
\hline FV-PTC & $1(6.7)$ & $4(26.7)$ & $2(13.3)$ & $8(53.3)$ & $0.280^{* *}$ \\
\hline TC-PTC & $1(12.5)$ & $0(0.0)$ & $2(25.0)$ & $5(62.5)$ & $0.592 * * *$ \\
\hline PD-PTC & $1(14.3)$ & $1(14.3)$ & $0(0.0)$ & $5(71.4)$ & \\
\hline FTC & $2(25.0)$ & $1(12.5)$ & $1(12.5)$ & $4(50.0)$ & \\
\hline \multicolumn{6}{|l|}{ Multifocality } \\
\hline Unifocal & $2(7.1)$ & $7(25.0)$ & $4(14.3)$ & $15(53.6)$ & 0.379 \\
\hline Multifocal & $5(17.9)$ & $7(25.0)$ & $1(3.6)$ & $15(53.6)$ & \\
\hline \multicolumn{6}{|l|}{ Concurrent CLT } \\
\hline Absent & $7(16.7)$ & $10(23.8)$ & $4(9.5)$ & $21(50.0)$ & 0.408 \\
\hline Present & $0(0.0)$ & $4(28.6)$ & $1(7.1)$ & $9(64.3)$ & \\
\hline $\begin{array}{l}\text { Tumor size, } \\
\text { Mean } \pm \text { SD }\end{array}$ & $4.6 \pm 2.9$ & $2.8 \pm 1.5$ & $3.7 \pm 1.9$ & $2.8 \pm 2.4$ & 0.185 \\
\hline \multicolumn{6}{|c|}{ LN metastasis at diagnosis } \\
\hline Absent & $3(9.1)$ & $6(18.2)$ & $2(6.1)$ & $22(66.7)$ & 0.163 \\
\hline Present & $4(17.4)$ & $8(34.8)$ & $3(13.0)$ & $8(34.8)$ & \\
\hline \multicolumn{6}{|c|}{ Distant metastasis at diagnosis } \\
\hline Absent & $3(6.4)$ & $12(25.5)$ & $4(8.5)$ & $28(59.6)$ & 0.013 \\
\hline Present & $4(44.5)$ & $2(22.2)$ & $1(11.1)$ & $2(22.2)$ & \\
\hline $\begin{array}{l}\text { RAI doses, } \\
\text { Mean } \pm \text { SD }\end{array}$ & $492.8 \pm 386.7$ & $247.6 \pm 173.1$ & $254.0 \pm 127.8$ & $230.0 \pm 261.5$ & 0.039 \\
\hline
\end{tabular}

Table 2. Comparison of ROR $\gamma t$ positivity and clinical and pathological features of aggressiveness of differentiated thyroid carcinomas. Abbreviations: SD, standard deviation; CLT, chronic lymphocytic thyroiditis; PTC, papillary thyroid carcinoma; FV-PTC, follicular variant of PTC; TC-PTC, tall cell variant of PTC; PDPTC, poorly differentiated, PTC; FTC, follicular thyroid carcinoma; RAI, radioactive iodine therapy cumulative doses. Note: ${ }^{a}$ The p-value expressed was obtained when the comparison was done considering the four different categories separated and not combined. *Comparison considering all different histologic subtypes (5 categories); ** comparison considering different subtype of PTC (4 categories); ***comparison considering PTC versus FTC.

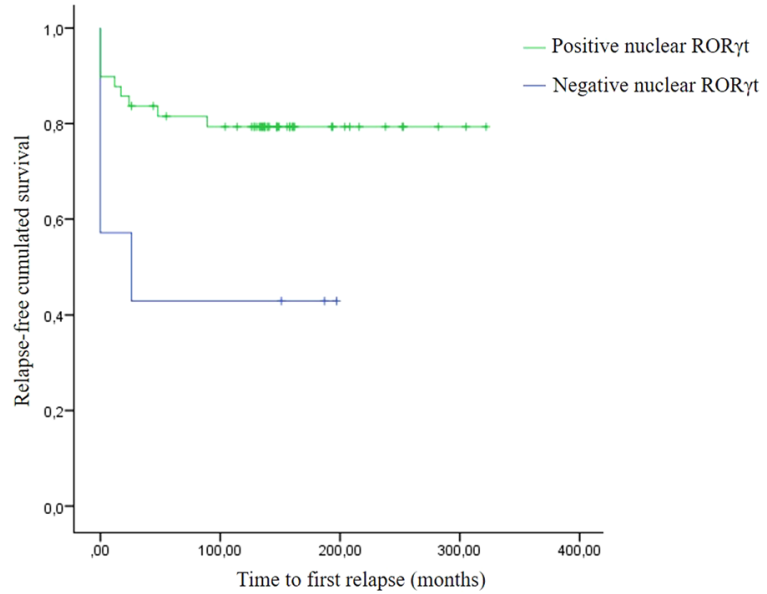

Figure 2. Kaplan-Meier curve shows that patients whose tumors were positive for ROR $\gamma$ t presented favorable outcome with higher relapse-free survival rate. 


\begin{tabular}{|c|c|c|c|}
\hline \multirow[b]{2}{*}{ Clinical feature } & \multicolumn{2}{|c|}{ Estimation of positivity for IL- $1 \beta$} & \multirow[b]{2}{*}{ p-value } \\
\hline & Negative & Positive & \\
\hline \multicolumn{4}{|l|}{ Gender } \\
\hline Male & $4(40.0)$ & $6(60.0)$ & 0.897 \\
\hline Female & $19(42.2)$ & $26(57.8)$ & \\
\hline \multicolumn{4}{|l|}{ Age at diagnosis } \\
\hline $45 \mathrm{yrs}$ or less & $11(42.3)$ & $15(57.7)$ & 0.944 \\
\hline more $45 \mathrm{yrs}$ & $12(41.4)$ & $17(58.6)$ & \\
\hline \multicolumn{4}{|l|}{ Histologic types } \\
\hline Classic PTC & $9(50.0)$ & $9(50.0)$ & $0.278^{*}$ \\
\hline FV-PTC & $5(33.3)$ & $10(66.7)$ & $0.268^{* *}$ \\
\hline TC-PTC & $2(25.0)$ & $6(75.0)$ & $0.297^{* * *}$ \\
\hline PD-PTC & $5(71.4)$ & $2(28.6)$ & \\
\hline FTC & $2(25.0)$ & $6(75.0)$ & \\
\hline \multicolumn{4}{|l|}{ Multifocality } \\
\hline Unifocal & $11(39.3)$ & $17(60.7)$ & 0.698 \\
\hline Multifocal & $12(44.4)$ & $15(55.6)$ & \\
\hline \multicolumn{4}{|l|}{ Concurrent CLT } \\
\hline Absent & $14(34.1)$ & 27 (65.9) & 0.048 \\
\hline Present & $9(64.3)$ & $5(35.7 \%)$ & \\
\hline Tumor size, Mean \pm SD & $2.4 \pm 2.0$ & $3.7 \pm 2.3$ & 0.032 \\
\hline \multicolumn{4}{|c|}{ LN metastasis at diagnosis } \\
\hline Absent & $15(45.5)$ & $18(54.5)$ & 0.503 \\
\hline Present & $8(36.4)$ & 14 (63.6) & \\
\hline \multicolumn{4}{|c|}{ Distant metastasis at diagnosis } \\
\hline Absent & $22(46.8)$ & $25(53.2)$ & 0.041 \\
\hline Present & $1(12.5)$ & $7(87.5)$ & \\
\hline RAI doses, Mean \pm SD & $178.2 \pm 206.0$ & $332.4 \pm 282.8$ & 0.004 \\
\hline
\end{tabular}

Table 3. Comparison of IL-1 $\beta$ positivity and clinical and pathological features of aggressiveness of differentiated thyroid carcinomas. Abbreviations: SD, standard deviation; CLT, chronic lymphocytic thyroiditis; PTC, papillary thyroid carcinoma; FV-PTC, follicular variant of PTC; TC-PTC, tall cell variant of PTC; PD-PTC, poorly differentiated, PTC; FTC, follicular thyroid carcinoma; RAI, radioactive iodine therapy cumulative doses. Note: *Comparison considering all different histologic subtypes ( 5 categories); **comparison considering different subtype of PTC (4 categories); *** comparison considering PTC versus FTC.

Th17-related cytokines frequently presented distant metastasis at diagnosis, suggesting that IL-1 $\beta$ and IL-23 may help to promote tumor invasion, while nuclear ROR $\gamma \mathrm{t}$ might inhibit tumor dissemination.

Zeng et al. obtained similar results ${ }^{17}$. Nuclear ROR $\gamma \mathrm{t}$ expression was inversely correlated to lymph node metastasis both in patients with PTC alone and in patients with concurrent Hashimoto's thyroiditis, corroborating our findings and suggesting that $\mathrm{ROR} \gamma \mathrm{t}$ might have an anti-tumor role in the tumor microenvironment. In fact, data from experimental investigation evidenced that ROR $\gamma \mathrm{t}$ agonist not only decreases the percentage of PD- $1+$ cells but also reduces the level of PD- 1 in individual immune cells ${ }^{32}$, suggesting that ROR $\gamma$ t may blunt $\mathrm{T}$ cell exhaustion, potentiate anti-tumor $\mathrm{T}$ cell response and diminish pro-tumor inflammatory response ${ }^{18,32}$. In addition, $\mathrm{Hu}$ et al. investigated tumor-bearing mice that received oral ROR $\gamma \mathrm{t}$ agonist compared to mice that received vehicle control. Mice that received oral $R O R \gamma t$ agonist presented an inhibition of tumor growth and longer survival compared to control ${ }^{32}$, reinforcing the idea that ROR $\gamma \mathrm{t}$ may be a potential therapeutic target for patients with cancer, including thyroid carcinoma.

We failed to correlate IL-17A with any clinical and pathological predictors of prognosis. On the contrary, Zeng et al. showed that the peripheral serum concentration of IL-17A was positively correlated with local invasion in PTC patients with no concurrent thyroiditis. When there was Hashimoto's thyroiditis concurrent to PTC, a negative correlation between IL-17A concentration and lymph node metastasis was observed. The reason why IL-17A accumulation behaves differently depending of the concurrence of Hashimoto's thyroiditis remains unclear. The concurrent autoimmunity may influence tumor microenvironment and help to sabotage the pro-tumor tendency of inflammatory response ${ }^{31,33}$. Carvalho et al. found a higher percentage of cells expressing IL-17 in patients with thyroid cancer $^{34}$. Additionally, IL-17 but not IL-23 expression was associated with recurrence/mortality, suggesting that the enrichment of IL-17A in tumor microenvironment may be related to aggressiveness in thyroid cancer $^{34}$. It is worthy to note that both pro- and anti-tumors role have been linked to IL-17A $\mathrm{A}^{35,36}$. These apparently conflicting data may be due to the multi-faceted immune responses associated with Th17 cells while IL-17A, as a unique cytokine, may reveal its effects depending on the tumor microenvironment and tumor histology $\mathrm{y}^{32,34,35}$. Interestingly, Carvalho et al. found a positive correlation between IL-17A and IL-23 expression. Likewise, we observed a tight correlation between IL-17A, IL-23, IL-1 $\beta$, all cytokines related to the Th17 arm of immune 


\begin{tabular}{|c|c|c|c|}
\hline \multirow[b]{2}{*}{ Clinical Feature } & \multicolumn{3}{|c|}{ Estimation of positivity for IL-23 } \\
\hline & Negative & Positive & p-value \\
\hline \multicolumn{4}{|l|}{ Gender } \\
\hline Male & $3(30.0)$ & $7(70.0)$ & 0.978 \\
\hline Female & $14(30.4)$ & $32(69.6)$ & \\
\hline \multicolumn{4}{|l|}{ Age at diagnosis } \\
\hline $45 \mathrm{yrs}$ or less & $9(33.3)$ & $18(66.7)$ & 0.640 \\
\hline more $45 \mathrm{yrs}$ & $8(27.6)$ & $21(72.4)$ & \\
\hline \multicolumn{4}{|l|}{ Histologic types } \\
\hline Classic PTC & $8(44.4)$ & $10(55.6)$ & 0.019* \\
\hline FV-PTC & $2(13.3)$ & $13(86.7)$ & $0.020^{* *}$ \\
\hline TC-PTC & $1(12.5)$ & $7(87.5)$ & $0.235 * * *$ \\
\hline PD-PTC & $5(71.4)$ & $2(28.6)$ & \\
\hline FTC & $1(12.5)$ & $7(87.5)$ & \\
\hline \multicolumn{4}{|l|}{ Multifocality } \\
\hline Unifocal & $7(25.0)$ & $21(75.0)$ & 0.383 \\
\hline Multifocal & $10(35.7)$ & $18(64.3)$ & \\
\hline \multicolumn{4}{|l|}{ Concurrent CLT } \\
\hline Absent & $11(26.2)$ & $31(73.8)$ & 0.240 \\
\hline Present & $6(42.9)$ & $8(57.1)$ & \\
\hline Tumor size, Mean \pm SD & $2.2 \pm 2.2$ & $3.6 \pm 2.2$ & 0.009 \\
\hline \multicolumn{4}{|c|}{ LN metastasis at diagnosis } \\
\hline Absent & $10(30.3)$ & $23(69.7)$ & 0.949 \\
\hline Present & $7(30.4)$ & $16(69.6)$ & \\
\hline \multicolumn{4}{|c|}{ Distant metastasis at diagnosis } \\
\hline Absent & $17(36.2)$ & $30(63.8)$ & 0.031 \\
\hline Present & $0(0.0)$ & $9(100.0)$ & \\
\hline RAI doses, Mean \pm SD & $158.8 \pm 153.3$ & $317.6 \pm 283.9$ & 0.037 \\
\hline
\end{tabular}

Table 4. Comparison of IL-23 positivity and clinical and pathological features of aggressiveness of differentiated thyroid carcinomas. Abbreviations: SD, standard deviation; CLT, chronic lymphocytic thyroiditis; PTC, papillary thyroid carcinoma; FV-PTC, follicular variant of PTC; TC-PTC, tall cell variant of PTC; PD-

PTC, poorly differentiated, PTC; FTC, follicular thyroid carcinoma; RAI, radioactive iodine therapy cumulative doses. Note: *comparison considering all different histologic subtypes ( 5 categories); **comparison considering different subtype of PTC (4 categories); ***comparison considering PTC versus FTC.

\begin{tabular}{|l|l|l|l|l|}
\hline \multirow{2}{*}{$\begin{array}{l}\text { Immune } \\
\text { markers }\end{array}$} & \multicolumn{4}{l}{ Immune markers } \\
\cline { 2 - 5 } & ROR $\gamma \mathbf{t}$ & IL-17A & IL-1 $\beta$ & IL-23 \\
\hline CD4 & 0.823 & 0.152 & 0.458 & 0.743 \\
\hline CD8 & 0.452 & 0.320 & $0.002^{\mathrm{d}}$ & $0.004^{\mathrm{d}}$ \\
\hline CD20 & 0.207 & 0.593 & 0.098 & 0.978 \\
\hline CD68 & 0.537 & 0.750 & 0.924 & 0.264 \\
\hline CD16 & $0.047^{\mathrm{i}}$ & 0.186 & $0.046^{\mathrm{d}}$ & $0.003^{\mathrm{d}}$ \\
\hline CD25 & 0.537 & 0.453 & 0.421 & 0.239 \\
\hline CD69 & 0.524 & 0.416 & 0.306 & 0.084 \\
\hline Granzyme B & 0.061 & 0.914 & 0.125 & 0.188 \\
\hline CD45RO & 0.521 & 0.759 & 0.795 & 0.354 \\
\hline CD134 & 0.380 & 0.702 & 0.358 & 0.110 \\
\hline COX2 & $0.024^{\mathrm{i}}$ & 0.169 & $<0.001^{\mathrm{d}}$ & $<0.001^{\mathrm{d}}$ \\
\hline IL-10 & 0.162 & 0.275 & $0.032^{\mathrm{d}}$ & 0.141 \\
\hline IL-23 & $0.043^{\mathrm{i}}$ & 0.308 & $<0.001^{\mathrm{d}}$ & \\
\hline IL-1 3 & $0.001^{\mathrm{i}}$ & 0.163 & & \\
\hline IL-17A & $0.035^{\mathrm{i}}$ & & & \\
\hline
\end{tabular}

Table 5. Association between immune markers to each other. Abbreviations: $d$, direct association; i, inverse association. Further molecular association between IL-10, Cox2, CD3, CD4, CD8, CD20, CD68, CD16, CD45RO, CD25, CD69 and Granzyme B was previously published by our group ${ }^{28,31,44}$. 

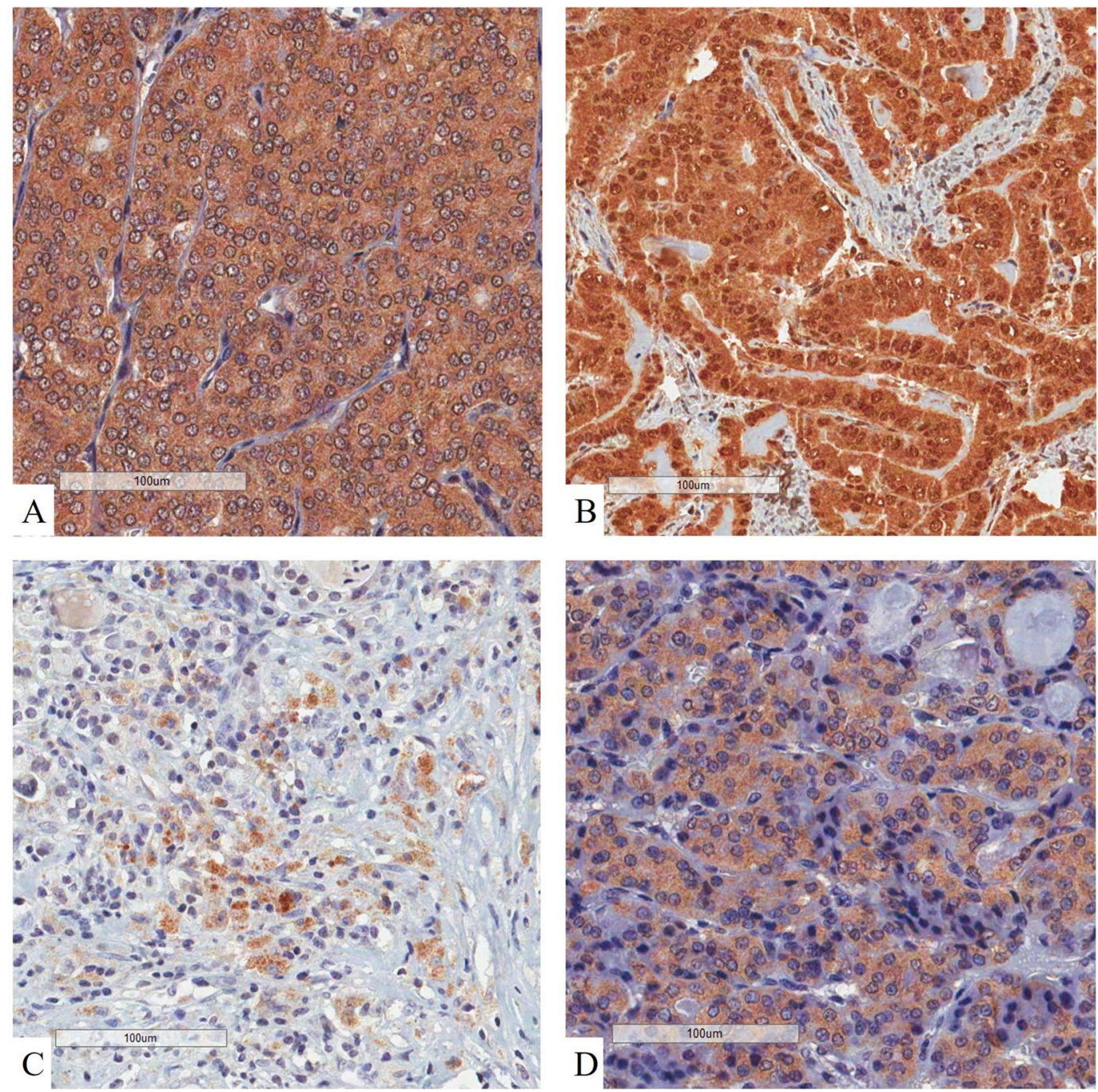

Figure 3. Immunohistochemistry of interleukins. (A) IL-1 $\beta$ presented a diffuse, homogeneous and cytoplasmic pattern of expression in thyroid cancer cells. (B) IL-10 was seen as strong brownish expression in both nuclei and cytoplasm of malignant cells. (C) IL-17A presented a focal cytoplasmic expression among thyroid cancer cells. (D) IL-23 expression was observed in cytoplasm of malignant cells. All panels evidenced that stromal structures (eg. collagen, colloid and vessels) lack expression of immune markers.

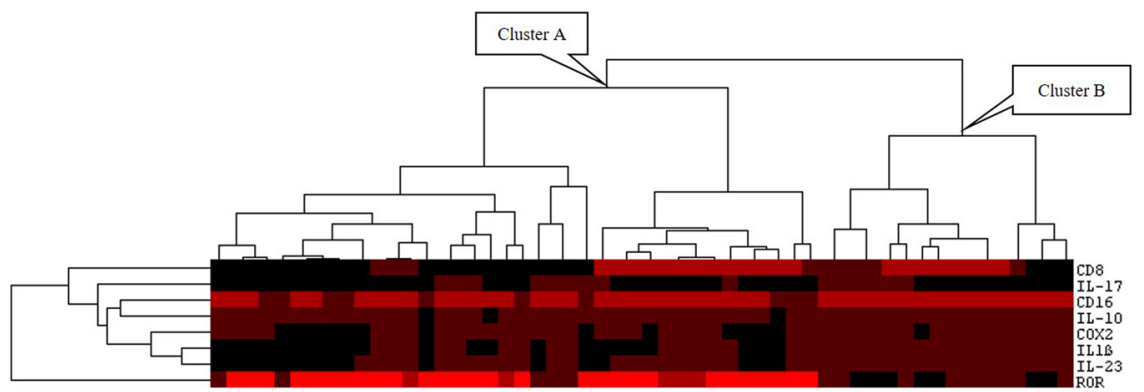

Figure 4. Dendrogram shows hierarchical cluster that classified patients in two different group. Group A represents patients who presented high expression of nuclear ROR $\gamma \mathrm{t}$ and tended to be scarce in proinflammatory immune markers. Group B represents patients whose tumor microenvironment accumulated proinflammatory markers and presented low expression of nuclear nuclear ROR $\gamma \mathrm{t}$. 


\begin{tabular}{|l|l|l|l|}
\hline \multirow{2}{*}{ Histologic types } & \multicolumn{4}{|l|}{ Cluster of molecular markers } \\
\cline { 2 - 4 } & $\mathbf{A}$ & $\mathbf{B}$ & p-value \\
\hline Classic PTC & $11(73.3)$ & $4(26.7)$ & $0.540^{*}$ \\
\hline FV-PTC & $11(78.6)$ & $3(21.4)$ & $0.859^{* *}$ \\
\hline TC-PTC & $7(87.5)$ & $1(12.5)$ & $0.134^{* * *}$ \\
\hline PD-PTC & $5(71.4)$ & $2(28.6)$ & \\
\hline FTC & $8(100.0)$ & $0(0.0)$ & \\
\hline
\end{tabular}

Table 6. Association between cluster of molecular markers and different tumor subtypes. Abbreviations: PTC, papillary thyroid carcinoma; FV-PTC, follicular variant of PTC; TC-PTC, tall cell variant of PTC; PD-PTC, poorly differentiated, PTC; FTC, follicular thyroid carcinoma. Note: *comparison considering all different histologic subtypes ( 5 categories); **comparison considering different subtype of PTC (4 categories); ***comparison considering PTC versus FTC.

response. More studies are warranted to unveil the specific role of IL-17A in tumor milieu of differentiated thyroid carcinoma.

Also, we demonstrated that patients whose tumors were positive for IL- $1 \beta$ required higher RAI cumulative doses, suggesting that IL- $1 \beta$ may be associated to RAI resistance. RAI gets into thyroid cells by the sodium iodide symporter (NIS). Spitzweg et al. investigated the modulation of NIS expression and NIS activity in thyroid cell lines ${ }^{37}$. Incubation of thyroid cells with IL- $1 \beta$ resulted in a $30 \%$ decrease of NIS mRNA steady-state levels ${ }^{37}$. IL- $1 \beta$ suppressed iodide accumulation by approximately $25 \%{ }^{37}$, favoring the idea that tumors that accumulate IL- $1 \beta$ may decrease NIS expression and activity, explaining why higher dose of RAI is required in those patients. Both autocrine and paracrine production of IL-1 $\beta$ may happen in tumor microenvironment and malignant thyroid follicular cells are capable of recognize bacterial lipopolysaccharide and elicit IL-1 $\beta$ production ${ }^{38}$. Many human cancers express IL-1 $\beta$ and its overproduction is associated with poor prognosis ${ }^{39,40}$. IL-1 $\beta$ polymorphisms that leads to overexpression of IL- $1 \beta$ might be a predictive factor for lymph node metastasis of PTC patients ${ }^{41}$, reinforcing the idea that IL-1 $\beta$ may help to promote tumor invasion and dissemination.

Our data have some limitations. The use of TMA could reduce the size of tumor samples, which may be seen as a limitation to its use. However, in a previous study on thyroid carcinoma, ROR mRNA was evaluated by in situ hybridization in TMA, and a good correlation was found with RT-PCR ${ }^{42}$. We believe that the results of the present study are representative of the real degree of ROR protein expression in PTC. We obtained the clinical and pathological information from the patient's charts retrospectively. Further prospective studies should dismiss this putative bias. In addition, we investigated protein expression of ROR $\gamma \mathrm{t}$ and Th17 related cytokines. We could not accurately assess how these molecules interact to each other in vitro and the undoubted role of the cytokines in the tumor microenvironment remains to be elucidated. In fact, most of literature investigated the nuclear ROR $\gamma$ t, IL-17A, IL-23 and IL-1 $\beta$ expression in lymphocytes, but not in epithelial cells. Herein, we reported the nuclear ROR $\gamma$ t, IL-17A, IL-23 and IL-1 $\beta$ in tumor cells of papillary thyroid cancer. Therefore, it is possible that the molecular relationships between ROR $\gamma$ t and IL-17A, IL-23 and IL-1 $\beta$ seen on lymphocytes cannot be extrapolated to tumor cells. In addition, we were not able to find any association between the investigated markers and patients' outcome. In fact, current therapy for DTC is very effective and, although excessive for most patients, it certainly contributes to the excellent prognosis of the patients, impairing long-term evaluation of the role of specific factors.

In summary, our data evidence that ROR $\gamma$ t is expressed in nuclei of PTC cells and this expression is associated with clinical and pathological features of favorable prognosis, suggesting that ROR $\gamma \mathrm{t}$ may favor anti-tumor immune response in the microenvironment of thyroid cancer. In fact, ROR $\gamma$ t was previously associated to favorable prognosis in renal and colorectal cancer ${ }^{43}$. On the contrary, IL-23 and IL-1 $\beta$ are associated to distant metastasis at diagnosis suggesting these cytokines may facilitate a pro-tumor inflammatory response engaged in tumor dissemination and aggressiveness.

Immunohistochemical expression of ROR $\gamma$ t, IL-23 and IL-1 $\beta$ can be easily accessed in routine pathology laboratories helping to predict the prognosis of patients with thyroid cancer and better individualize their clinical management. A personalized individual clinical approach is of utmost need in order to consider the effect of different immune markers and their relationship.

Received: 9 December 2019; Accepted: 7 February 2020;

Published online: 05 March 2020

\section{References}

1. American Cancer Society. Key statistics for thyroid cancer, https://www.cancer.org/cancer/thyroid-cancer/about/key-statistics.html (2019).

2. La Vecchia, C. et al. Thyroid cancer mortality and incidence: a global overview. Int. J. Cancer. 136, 2187-2195 (2015).

3. Ahn, H. S., Kim, H. J. \& Welch, H. G. Korea's thyroid-cancer "epidemic"-screening and overdiagnosis. N. Engl. J. Med. 371, 1765-1767 (2014).

4. Dal Maso, L. et al. The impact of overdiagnosis on thyroid cancer epidemic in Italy,1998-2012. Eur. J. Cancer. 94, 6-15 (2018).

5. Nikiforov, Y. E. Role of molecular markers in thyroid nodule management: then and now. Endocr. Pract. 23, 979-988 (2017).

6. Liotti, F., Prevete, N., Vecchio, G. \& Melillo, R. M. Recent advances in understanding immune phenotypes of thyroid carcinomas: prognostication and emerging therapies. F1000 Research. 8, 227 (2019).

7. Ferrari, S. M. et al. Immune and Inflammatory Cells in Thyroid Cancer Microenvironment. Int. J. Mol. Sci. 20, E4413 (2019). 
8. Yapa, S., Mulla, O., Green, V., England, J. \& Greenman, J. The Role of Chemokines in Thyroid Carcinoma. Thyroid. 27, 1347-1359 (2017).

9. Rotondi, M., Coperchini, F., Latrofa, F. \& Chiovato, L. Role of Chemokines in Thyroid Cancer Microenvironment: Is CXCL8 the Main Player? Front. Endocrinol. 9, 314 (2018).

10. Coperchini, F., Croce, L., Marinò, M., Chiovato, L. \& Rotondi, M. Role of chemokine receptors in thyroid cancer and immunotherapy. Endocr. Relat. Cancer. 26, R465-R478 (2019).

11. Cunha, L. L., Marcello, M. A. \& Ward, L. S. The role of the inflammatory microenvironment in thyroid carcinogenesis. Endocr. Relat. Cancer. 21, R85-R103 (2014).

12. Marcello, M. A. et al. The influence of the environment on the development of thyroid tumors: a new appraisal. Endocr. Relat. Cancer. 21, T235-254 (2014).

13. Eberl, G. \& Littman, D. R. The role of the nuclear hormone receptor RORgammat in the development of lymph nodes and Peyer's patches. Immunol. Rev. 195, 81-90 (2003).

14. Ivanov, I. I. et al. The orphan nuclear receptor RORgammat directs the differentiation program of proinflammatory IL-17+ Thelper cells. Cell. 126, 1121-1133 (2006).

15. He, Y. W., Deftos, M. L., Ojala, E. W. \& Bevan, M. J. RORgammat, a novel isoform of an orphan receptor, negatively regulates Fas ligand expression and IL-2 production in T cells. Immunity. 9, 797-806 (1998).

16. Irshad, S. et al. ROR $\gamma t+$ innate lymphoid cells promote lymph node metastasis of breast cancers. Cancer Res. 77, 1083-1096 (2017).

17. Zeng, R. et al. Positive effect of ROR $\gamma \mathrm{t}$ on the prognosis of thyroid papillary carcinoma patients combined with Hashimoto's thyroiditis. Am. J. Transl. Res. 10, 3011-3024 (2018).

18. Chang, M. R. et al. Synthetic ROR $\gamma$ t Agonists Enhance Protective Immunity. ACS Chem. Biol. 11, 1012-1018 (2016).

19. Chang, M. R., Goswami, D., Mercer, B. A. \& Griffin, P. R. The therapeutic potential of ROR $\gamma$ modulators in the treatment of human disease. J. Exp. Pharmacol. 4, 141-148 (2012).

20. Fauber, B. P. \& Magnuson, S. Modulators of the nuclear receptor retinoic acid receptor-related orphan receptor- $\gamma$ (ROR $\gamma$ or RORc). J. Med. Chem. 57, 5871-5892 (2014).

21. clinicaltrials.gov. Study of LYC-55716 in Adult Subjects With Locally Advanced or Metastatic Cancer, https:/clinicaltrials.gov/ct2/ show/NCT02929862 (2019).

22. corp. L. Lycera Announces Initiation of Phase $1 \mathrm{~b}$ Study of Novel Immuno-Oncology Candidate LYC-55716 in Combination with Pembrolizumab, https://www.prnewswire.com/news-releases/lycera-announces-initiation-of-phase-1b-study-of-novel-immunooncology-candidate-lyc-55716-in-combination-with-pembrolizumab-300577211.html (2019).

23. Kasagi, K. et al. Clinical significance of measurements of antithyroid antibodies in the diagnosis of Hashimoto's thyroiditis: comparison with histological findings. Thyroid. 6, 445-450 (1996).

24. Janjua, N. \& Wreesmann, V. B. Aggressive differentiated thyroid cancer. Eur. J. Surg. Oncol. 44, 367-377 (2018)

25. Dettmer, M. et al. Poorly differentiated thyroid carcinomas: how much poorly differentiated is needed? Am. J. Surg. Pathol. 35, 1866-72 (2011)

26. Nambiar, A., Pv, S., Susheelan, V. \& Kuriakose, M. A. The concepts in poorly differentiated carcinoma of the thyroid: a review article. J. Surg. Oncol. 103, 818-821 (2011).

27. Haugen, B. R. et al. American Thyroid Association Management Guidelines for Adult Patients with Thyroid Nodules and Differentiated Thyroid Cancer: The American Thyroid Association Guidelines Task Force on Thyroid Nodules and Differentiated Thyroid Cancer. Thyroid. 26, 1-133 (2015).

28. Cunha, L. L. et al. Interleukin 10 expression is related to aggressiveness and poor prognosis of patients with thyroid cancer. Cancer Immunol. Immunother. 66, 141-148 (2017).

29. Cunha, L. L. et al. Foxp3 expression is associated with aggressiveness in differentiated thyroid carcinomas. Clinics. 67, 483-488 (2012).

30. Morari, E. C. et al. Muc-1 expression may help characterize thyroid nodules but does not predict patients' outcome. Endocr. Pathol. 21, 242-249 (2010).

31. Cunha, L. L. et al. Infiltration of a mixture of different immune cells may be related to molecular profile of differentiated thyroid cancer. Endocr. Relat. Cancer. 19, L31-36 (2012).

32. Hu, X. et al. Synthetic ROR $\gamma$ agonists regulate multiple pathways to enhance antitumor immunity. Oncoimmunology. 5, e1254854 (2016).

33. Cunha, L. L., Ferreira, R. C., Marcello, M. A., Vassallo, J. \& Ward, L. S. Clinical and pathological implications of concurrent autoimmune thyroid disorders and papillary thyroid cancer. J. Thyroid. Res. 17, 387062 (2011)

34. Carvalho, D. F. G. et al. High IL-17 expression is associated with an unfavorable prognosis in thyroid cancer. Oncol. Lett. 13, 1925-1931 (2017).

35. Zou, W. \& Restifo, N. P. T(H)17 cells in tumour immunity and immunotherapy. Nat. Rev. Immunol. 10, 248-256 (2010).

36. Bailey, S. R. et al. Th17 cells in cancer: the ultimate identity crisis. Front. Immunol. 5, 276 (2014).

37. Spitzweg, C., Joba, W., Morris, J. C. \& Heufelder, A. E. Regulation of sodium iodide symporter gene expression in FRTL-5 rat thyroid cells. Thyroid. 9, 821-830 (1999).

38. Plantinga, T. S. et al. Association of NF- $\kappa$ B polymorphisms with clinical outcome of non-medullary thyroid carcinoma. Endocr. Relat. Cancer. 24, 307-318 (2017).

39. Mukhopadhyay, P. et al. The cyclin-dependent kinase 2 inhibitor down-regulates interleukin-1beta-mediated induction of cyclooxygenase-2 expression in human lung carcinoma cells. Cancer Res. 66, 1758-1766 (2006).

40. Maihöfner, C. et al. Expression of cyclooxygenase-2 parallels expression of interleukin-1beta, interleukin-6 and NF-kappaB in human colorectal cancer. Carcinogenesis. 24, 665-671 (2003).

41. Ban, J. Y., Kim, M. K., Park, S. W. \& Kwon, K. H. Interleukin-1 beta polymorphisms are associated with lymph node metastasis in Korean patients with papillary thyroid carcinoma. Immunol. Invest. 41, 888-905 (2012).

42. Zhang, R., Hardin, H., Huang, W., Buehler, D. \& Lloyd, R. V. Long Non-coding RNA Linc-ROR Is Upregulated in Papillary Thyroid Carcinoma. Endocr. Pathol. 29, 1-8 (2018).

43. Atlas, H. P. Expression on RORC in cancer, https://www.proteinatlas.org/ENSG00000143365-RORC/pathology (2019).

44. Cunha, L. L. et al. CD8 + tumour-infiltrating lymphocytes and COX2 expression may predict relapse in differentiated thyroid cancer. Clin. Endocrinol. 83, 246-253 (2015).

\section{Author contributions}

Lucas Leite Cunha: Conception and design, critical review of the literature and data, data analysis, composition of the manuscript and final approval. Elaine Cristina Morari: Design, critical review of the literature and data, composition of the manuscript and final approval. Suely Nonogaki: Design, critical review of the literature and data, composition of the manuscript and final approval. Natassia Elena Bufalo: Design, critical review of the literature and data, composition of the manuscript and final approval. Ligia Vera Montalli da Assumpção: Design, critical review of the data, data collection, composition of the manuscript and final approval. Fernando Augusto Soares: Design, critical review of the literature and data, composition of the manuscript and final approval. José 
Vassallo: Design, critical review of the literature and data, composition of the manuscript and final approval. Laura Sterian Ward: Design, selection of notable articles for review, critical review of the literature and data, composition of the manuscript, clinical and translational orientation and final approval.

\section{Competing interests}

The authors declare no competing interests.

\section{Additional information}

Correspondence and requests for materials should be addressed to L.S.W.

Reprints and permissions information is available at www.nature.com/reprints.

Publisher's note Springer Nature remains neutral with regard to jurisdictional claims in published maps and institutional affiliations.

(c) (i) Open Access This article is licensed under a Creative Commons Attribution 4.0 International License, which permits use, sharing, adaptation, distribution and reproduction in any medium or format, as long as you give appropriate credit to the original author(s) and the source, provide a link to the Creative Commons license, and indicate if changes were made. The images or other third party material in this article are included in the article's Creative Commons license, unless indicated otherwise in a credit line to the material. If material is not included in the article's Creative Commons license and your intended use is not permitted by statutory regulation or exceeds the permitted use, you will need to obtain permission directly from the copyright holder. To view a copy of this license, visit http://creativecommons.org/licenses/by/4.0/.

(C) The Author(s) 2020 